\title{
EXTREME POINTS AND SATURATED POLYNOMIALS
}

\author{
GREG KNESE
}

\begin{abstract}
We consider the problem of characterizing the extreme points of the set of analytic functions $f$ on the bidisk with positive real part and $f(0)=1$. If one restricts to those $f$ whose Cayley transform is a rational inner function, one gets a more tractable problem. We construct families of such $f$ that are extreme points and conjecture that these are all such extreme points. These extreme points are constructed from polynomials dubbed $\mathbb{T}^{2}$-saturated, which roughly speaking means they have no zeros in the bidisk and as many zeros as possible on the boundary without having infinitely many zeros.
\end{abstract}

\section{Contents}

1. Introduction

2. Proof of McDonald's characterization of faces

3. Preliminaries for Theorems 1.5 and 1.6

4. Completion of the proof of Theorem 1.5

5. Completion of the proof of Theorem 1.6

6. Degree $(1,1)$ polynomials

7. Example of a non-saturated polynomial 16

8. Integrability of perturbations 17

9. Determinantal representations and transfer function realizations 19

10. Acknowledgments 22

References

\section{INTRODUCTION}

Let $P R P_{d}$ denote the set of holomorphic functions $f: \mathbb{D}^{d} \rightarrow R H P$ such that $f(0)=1$. Here $\mathbb{D}^{d}$ is the $d$-dimensional unit polydisk in $\mathbb{C}^{d}$ and $R H P$ is the right half plane in $\mathbb{C}$.

The set $P R P_{d}$ is a normal family and a convex set. Rudin posed the following natural question in his 1970 ICM address [16].

What are the extreme points of $P R P_{d}$ ?

Date: March 2, 2017.

2010 Mathematics Subject Classification. Primary: 32A10, Secondary 46A55, 47A57.

Key words and phrases. extreme points, polydisk, polydisc, bidisk, bidisc, holomorphic, analytic, intersection multiplicity, two-torus, determinantal representations, transfer functions, bounded analytic functions, inner functions, rational inner functions, stable polynomial, scattering stable polynomial.

Partially supported by NSF grant DMS-1363239. 
A complete answer is known only in the case $d=1$. The extreme points of $P R P_{1}$ are the functions

$$
z \mapsto \frac{1+\bar{\alpha} z}{1-\bar{\alpha} z}
$$

where $\alpha \in \mathbb{T}$, the unit circle. Remarkably, this fact together with the Krein-Milman theorem suffices to prove the Herglotz representation theorem for elements of $P R P_{1}$ : for every $f \in$ $P R P_{1}$ there is a unique probability measure $\mu$ on $\mathbb{T}$ such that

$$
f(z)=\int_{\mathbb{T}} \frac{1+\bar{\zeta} z}{1-\bar{\zeta} z} d \mu(\zeta) .
$$

In turn, this fact can be used to prove a variety of spectral theorems.

There is no known characterization of the extreme points of $P R P_{d}$ for $d>1$ and we would guess there is not a simple characterization. Every $f \in P R P_{d}$ has a Poisson type representation

$$
\operatorname{Re} f(z)=\int_{\mathbb{T}^{d}} P_{z_{1}} P_{z_{2}} \cdots P_{z_{d}} d \mu
$$

where $P_{w}(\zeta)=\frac{1-|w|^{2}}{|1-\bar{w} \zeta|^{2}}$ is the Poisson kernel and $\mu$ is a probability measure. However, when $d>1, \mu$ cannot be an arbitrary probability measure; it must satisfy additional moment conditions since $f$ is analytic. Specifically, we must have $\operatorname{supp}(\hat{\mu}) \subset \mathbb{Z}_{+}^{d} \cup \mathbb{Z}_{-}^{d}$ where $\mathbb{Z}_{+}$ (resp. $\mathbb{Z}_{-}$) denotes the set of non-negative (resp. non-positive) integers. One might expect that extreme points of $P R P_{d}$ would be represented via measures with "small" support but McDonald has constructed an extreme point whose boundary measure is absolutely continuous with respect to Lebesgue measure on $\mathbb{T}^{d}$ (see [13]). Not all of the known results are negative in spirit however.

Forelli has a useful necessary condition for $f \in P R P_{d}$ to be an extreme point [5]. Let $S_{d}$ denote the Schur class of the polydisk; the set of holomorphic functions $F: \mathbb{D}^{d} \rightarrow \overline{\mathbb{D}}$. First, via a Cayley transform we may write

$$
f=\frac{1+F}{1-F}
$$

where $F \in S_{d}$ and $F(0)=0$. Forelli has proven that if $F$ is reducible in the sense that $F=G H$ with non-constant $G, H \in S_{d}$ with $G(0)=0$ (but with no assumption on $H(0)$ ), then $f$ is not an extreme point of $P R P_{d}$. Forelli's approach actually gives an elementary proof of the characterization of extreme points for the case $d=1$.

Since the general problem of characterizing extreme points of $P R P_{d}$ seems difficult, it seems reasonable to restrict the problem to a more tractable subclass of functions. We shall examine the class

$$
P R P R_{d}=\left\{f \in P R P_{d}: F=\frac{f-1}{f+1} \text { is a rational inner function }\right\} .
$$

Inner means for almost every $z \in \mathbb{T}^{d},|F(r z)| \rightarrow 1$ as $r \nearrow 1$. This implies $\Re f(r z) \rightarrow 0$ at least away from $z \in \mathbb{T}^{d}$ such that $F(r z) \rightarrow 1$. This class, while very special, is also dense in $P R P_{d}$ using the topology of local uniform convergence. (Rudin [15] proves that rational inner functions are dense in $S_{d}$ and it then follows that $P R P R_{d}$ is dense in $P R P_{d}$.) Thus, we shall modify our opening question from Rudin.

Which $f \in P R P R_{d}$ are extreme points of $P R P_{d}$ ? 
It is proven in Rudin [15] that rational inner functions are of the form

$$
F(z)=\frac{\tilde{p}(z)}{p(z)}
$$

where $p \in \mathbb{C}\left[z_{1}, \ldots, z_{d}\right]$

(1) has no zeros in $\mathbb{D}^{d}$,

(2) multidegree at most $n=\left(n_{1}, \ldots, n_{d}\right)$ (the degree in $z_{j}$ is at most $\left.n_{j}\right)$, and

(3) the reflection of $p$ given by

$$
\tilde{p}(z)=z_{1}^{n_{1}} \cdots z_{d}^{n_{d}} \overline{p\left(1 / \bar{z}_{1}, \ldots, 1 / \bar{z}_{d}\right)}
$$

has no factors in common with $p$.

We say multidegree "at most" above to allow for $\tilde{p}$ to have a monomial factor. Since $p(0) \neq 0$, we get that $\tilde{p}$ has multidegree exactly $n$. For this reason it often makes more sense to refer to the multidegree of $\tilde{p}$.

Definition 1.1. We say $p \in \mathbb{C}\left[z_{1}, \ldots, z_{d}\right]$ is scattering stable if it satisfies conditions (1)-(3) above for $n$ equal to the multidegree of $p$.

Some simple examples of rational inner functions are

$$
G_{1}=\frac{3 z_{1} z_{2}-z_{1}-z_{2}}{3-z_{1}-z_{2}} \quad G_{2}=\frac{2 z_{1} z_{2}-z_{1}-z_{1}}{2-z_{1}-z_{2}}
$$

and corresponding elements of $P R P_{2}$ are

$$
g_{1}=\frac{3-2\left(z_{1}+z_{2}\right)+3 z_{1} z_{2}}{3\left(1-z_{1} z_{2}\right)} \quad g_{2}=\frac{\left(1-z_{1}\right)\left(1-z_{2}\right)}{1-z_{1} z_{2}} .
$$

One way to analyze a convex set is to examine its faces. Given an element $x$ of a convex set $S$, its face $\mathcal{F}(x)$ is the union of all line segments $L$ in $S$ with $x$ in the interior of $L$. We set $\mathcal{F}(x)=\{x\}$ when $x$ is an extreme point. McDonald has characterized the faces of $f \in P R P R_{d}$ as elements of the convex set $P R P_{d}$.

Theorem 1.2 (McDonald [12]). Let $f \in P R P R_{d}$ which we write as $f=\frac{1+F}{1-F}=\frac{p+\tilde{p}}{p-\tilde{p}}$ where $F=\frac{\tilde{p}}{p}$ is a rational inner function, written as above with $\tilde{p}(0)=0$ and multidegree $n$. Then, every element of the face $\mathcal{F}(f)$ of $f$ in $P R P_{d}$ is of the form

$$
\frac{p+\tilde{p}+2 v}{p-\tilde{p}}
$$

where $v \in \mathbb{C}\left[z_{1}, \ldots, z_{d}\right]$ has multidegree at most $n$, satisfies $v=\tilde{v}, v(0)=0$, and $p+v$ and $p$ - av have no zeros in $\mathbb{D}^{d}$ for some $a>0$. The face $\mathcal{F}(f)$ has real dimension at most $\prod\left(n_{j}+1\right)-2$.

The condition with $a$ is just to make $f$ in the interior of an appropriate line segment. This theorem does not solve our problem because the condition that $p+v$ and $p-a v$ have no zeros in $\mathbb{D}^{d}$ is difficult to work with. Nevertheless, the theorem certainly provides a useful reduction. Indeed, it shows that $\mathcal{F}(f)$ does not depend on the choice of underlying convex set $\left(P R P_{d}\right.$ versus $\left.P R P R_{d}\right)$.

The original proof of McDonald involves one variable slices and the Herglotz representation theorem. We shall give a very similar proof that uses only complex analysis (i.e. no measure theory). The number $\prod\left(n_{j}+1\right)-2$ simply counts the real-linear dimension of the set of $v$ 
of multidegree at most $n$ satisfying $v(0)=0$ and $v=\tilde{v}$. In particular, if $p$ has no zeros in $\overline{\mathbb{D}}^{d}$, then this dimension is exactly $\prod\left(n_{j}+1\right)-2$ since $p \pm t v$ will have no zeros in $\overline{\mathbb{D}}^{d}$ for $|t|$ small enough. Thus, if $p$ has no zeros in $\overline{\mathbb{D}}^{d}$ then $f=(p+\tilde{p}) /(p-\tilde{p})$ is never an extreme point unless $p$ depends on only one variable.

For example, the function $g_{1}$ in (1.3) is not extreme and we can perturb $p_{1}=3-z_{1}-z_{2}$ using any $v=a z_{1}+\bar{a} z_{2}$ with small enough $a \in \mathbb{C}$. In fact, $p_{1}+a z_{1}+\bar{a} z_{2}$ has no zeros in $\mathbb{D}^{2}$ if and only if $|1-a| \leq 3 / 2$.

We have been able to prove the following add-on to McDonald's theorem, which also says that the dimension of $\mathcal{F}(f)$ is maximal if and only if $p$ has no zeros in $\overline{\mathbb{D}}^{d}$.

Theorem 1.3. Assume the setup of Theorem 1.2. If $p$ vanishes at $\zeta \in \mathbb{T}^{d}$ to order $M$, then $v$ vanishes at least to order $M$ at $\zeta$.

We can also say something about the homogeneous expansion of $v$. See Theorem 2.2 for details.

Unfortunately, Theorem 1.3 has limitations because order of vanishing is a crude way to count zeros in this setting. Consider the example $g_{2}$ above. Since $p_{2}=2-z_{1}-z_{2}$ has a zero at $(1,1)$, so must any valid perturbation $v=a z_{1}+\bar{a} z_{2}$. This implies $a=i r$ for some $r \in \mathbb{R}$. It turns out $g_{2}$ is actually an extreme point, but it takes additional work to prove this (i.e. to show $r=0$ ).

Our main goal is to prove a theorem which describes a number of extreme points of $P R P_{d}$ coming from $P R P R_{d}$ in the case $d=2$. The denominators of the $F$ 's appearing in these extreme points have a special property.

Definition 1.4. Let $p \in \mathbb{C}\left[z_{1}, z_{2}\right]$ be scattering stable and of bidegree $\left(n_{1}, n_{2}\right)$. We say $p$ is $\mathbb{T}^{2}$-saturated if $p$ and $\tilde{p}$ have $2 n_{1} n_{2}$ common zeros on $\mathbb{T}^{2}$.

The common zeros of $p$ and $\tilde{p}$ are counted with multiplicities as in Bézout's theorem. The number $2 n_{1} n_{2}$ counts all of the common zeros of $p$ and $\tilde{p}$ on $\mathbb{C}_{\infty} \times \mathbb{C}_{\infty}$. See [3, 4] for more details about intersection multiplicities.

The class of $\mathbb{T}^{2}$-saturated polynomials is interesting in its own right. In the paper [10], we gave two related characterizations of $\mathbb{T}^{2}$-saturated polynomials. One relates to a sumsof-squares formula for scattering stable polynomials. If $p \in \mathbb{C}\left[z_{1}, z_{2}\right]$ is scattering stable of bidegree $\left(n_{1}, n_{2}\right)$ then

$$
|p|^{2}-|\tilde{p}|^{2}=\left(1-\left|z_{1}\right|^{2}\right) S O S_{1}+\left(1-\left|z_{2}\right|^{2}\right) S O S_{2}
$$

where $S O S_{j}$ is sum of squared moduli of $n_{j}$ polynomials. A scattering stable polynomial $p$ is $\mathbb{T}^{2}$-saturated if and only if the sums of squares terms $S O S_{1}, S O S_{2}$ are unique (see Corollary 13.6 of [10]).

A second characterization says that a scattering stable polynomial $p$ is $\mathbb{T}^{2}$-saturated if and only if

$$
\int_{\mathbb{T}^{2}}\left|\frac{f}{p}\right|^{2} d \sigma=\infty
$$

for all nonzero $f \in \mathbb{C}\left[z_{1}, z_{2}\right]$ satisfying $\operatorname{deg}_{j} f<\operatorname{deg}_{j} p$ for $j=1,2$ (see Corollary 6.5 of [10]). Thus, $\mathbb{T}^{2}$-saturated polynomials have so many boundary zeros that lower degree polynomials cannot match them (in the above sense). 
A simple example of a $\mathbb{T}^{2}$-saturated polynomial is $p=2-z_{1}-z_{2}$. Note $p$ and $\tilde{p}$ share a single zero $(1,1)$ on $\mathbb{T}^{2}$ but it has multiplicity 2 . This can be computed using the resultant of $p$ and $\tilde{p}$ with respect to $z_{2}$.

Our main theorem suggests a third characterization of $\mathbb{T}^{2}$-saturated polynomials and constructs a family of extreme points in $P R P_{2}$.

Theorem 1.5. Let $p \in \mathbb{C}\left[z_{1}, z_{2}\right]$ be scattering stable and $\tilde{p}(0,0)=0$. Let $f=\frac{p+\tilde{p}}{p-\tilde{p}}$. If $p$ is $\mathbb{T}^{2}$-saturated and $p-\tilde{p}$ is irreducible, then $f$ is an extreme point of $P R P_{2}$.

For example, one can conclude the example $g_{2}$ is an extreme point simply by noticing $p=2-z_{1}-z_{2}$ is $\mathbb{T}^{2}$-saturated and $p-\tilde{p}=2\left(1-z_{1} z_{2}\right)$ is irreducible. On the other hand, $p$ being $\mathbb{T}^{2}$-saturated is not sufficient for $f$ to be extreme as the example

$$
\frac{1-G_{2}}{1+G_{2}}=\frac{\left(1-z_{1} z_{2}\right)}{\left(1-z_{1}\right)\left(1-z_{2}\right)}=\frac{1}{2}\left(\frac{1+z_{1}}{1-z_{1}}+\frac{1+z_{2}}{1-z_{2}}\right)
$$

shows.

Along the path to proving Theorem 1.5 we have been able to prove a more refined version of Theorem 1.3. Given two polynomials $p, q \in \mathbb{C}\left[z_{1}, z_{2}\right]$ with a common zero $\zeta$, we let $I_{\zeta}(p, q)$ denote the intersection multiplicity of $p, q$ at $\zeta$.

Theorem 1.6. Let $f=\frac{p+\tilde{p}}{p-\tilde{p}} \in P R P R_{2}$ where $p \in \mathbb{C}\left[z_{1}, z_{2}\right]$ is scattering stable. Suppose

$$
g=f+2 \frac{v}{p-\tilde{p}}
$$

belongs to the face of $f$ as in Theorem 1.2. Suppose $p(\zeta)=0$ for some $\zeta \in \mathbb{T}^{2}$. Then,

$$
I_{\zeta}(p, \tilde{p}) \leq I_{\zeta}(p+v, \tilde{p}+v) .
$$

We have been unable to prove the following.

Conjecture 1.7. If $p \in \mathbb{C}\left[z_{1}, z_{2}\right]$ is scattering stable, $\tilde{p}(0,0)=0$, and $f=\frac{p+\tilde{p}}{p-\tilde{p}}$ is an extreme point of $P R P_{2}$, then $p$ is $\mathbb{T}^{2}$-saturated and $p-\tilde{p}$ is irreducible.

A very modest piece of evidence is the following. See section 6 .

Theorem 1.8. Conjecture 1.7 holds if $p$ has bidegree $(1,1)$.

The reason Theorem 1.8 works out is that if $f \in P R P R_{2}$ is extreme and depends on both variables, then the associated $p$ must have at least one zero on $\mathbb{T}^{2}$. In the case of degree $(1,1)$ polynomials, a single zero implies saturation. In Section 7 we present an example of a non-saturated scattering stable polynomial with a zero on $\mathbb{T}^{2}$ and we show that the associated $f$ is not extreme. This at least shows that $\mathbb{T}^{2}$-saturated should not be replaced with $p$ having at least one zero on $\mathbb{T}^{2}$.

One final piece of evidence in favor of Conjecture 1.7 is the following.

Theorem 1.9. Suppose $p \in \mathbb{C}\left[z_{1}, z_{2}\right]$ is scattering stable with $\tilde{p}(0,0)=0$.

If there exists $v \in \mathbb{C}\left[z_{1}, z_{2}\right]$ such that $\operatorname{deg} v \leq \operatorname{deg} \tilde{p}, v(0,0)=0, v=\tilde{v}$, and $p \pm v$ have no zeros in $\mathbb{D}^{2}$, then $v / p \in L^{2}\left(\mathbb{T}^{2}\right)$.

Going in the other direction, if $p$ is not $\mathbb{T}^{2}$-saturated then there exists nonzero $v \in \mathbb{C}\left[z_{1}, z_{2}\right]$ such that $v / p \in L^{2}\left(\mathbb{T}^{2}\right), \operatorname{deg} v \leq \operatorname{deg} \tilde{p}, v=\tilde{v}$, and $v(0,0)=0$. 
Of course the key thing missing in the last statement is $p \pm t v$ have no zeros in $\mathbb{D}^{2}$ for some small $t>0$. This theorem requires some machinery from [10].

In Section 9 we discuss some connections to determinantal representations for stable polynomials and transfer function representations for analytic functions. In particular, we point out that saturated polynomials $p$ possess symmetric, contractive determinantal representations, and the corresponding $F=\tilde{p} / p$ possess symmetric unitary transfer function realizations. These notions are all defined in Section 9.

\section{Proof of McDonald's Characterization of FaCES}

In this section we will give a slightly more elementary proof of McDonald's characterization of faces (Theorem 1.2) for $f=\frac{1+F}{1-F}$ where $F$ is rational inner. It is essentially the same proof except we avoid measure theory and gain a little more information and the face of $f$.

We first tackle the one variable case where $F$ is a Blaschke product which we may write as $F=\frac{\tilde{p}}{p}$ for a polynomial $p \in \mathbb{C}[z]$ having no zeros in $\overline{\mathbb{D}}$ (because zeros on $\mathbb{T}$ get cancelled out) and with $\tilde{p}(0)=0$. Then, $f=\frac{p+\tilde{p}}{p-\tilde{p}}$.

Let $f_{1}$ be in the face $\mathcal{F}(f)$ corresponding to $f$. This means there exist $a>0$ and analytic $H$ such that $f_{t}=f+t H \in P R P_{1}$ for $t \in[-a, 1]$. It will be convenient later to write $H=\frac{2 v}{p-\tilde{p}}$ for some holomorphic function $v$. Division by $p-\tilde{p}$ is no loss of generality since this function is non-vanishing in $\mathbb{D}$ (indeed, by the maximum principle $F \neq 1$ in $\mathbb{D}$ ). Note that

$$
f_{1}=\frac{p+\tilde{p}}{p-\tilde{p}}+\frac{2 v}{p-\tilde{p}}=\frac{1+\frac{\tilde{p}+v}{p+v}}{1-\frac{\tilde{p}+v}{p+v}} .
$$

Let $A=\left\{a_{1}, \ldots, a_{n}\right\}$ be the set of zeros of $p-\tilde{p}$ on $\mathbb{T}$. Since $\Re f=\frac{a}{a+1} \Re f_{1}+\frac{1}{a+1} \Re f_{-a} \geq 0$ and since $\Re f=\frac{|p|^{2}-|\tilde{p}|^{2}}{|p-\tilde{p}|^{2}}=0$ for $z \in \mathbb{T} \backslash A$ we see that for all $t \in[-a, 1]$

$$
\Re f_{t}(z) \rightarrow 0
$$

as $z \rightarrow \mathbb{T} \backslash A$.

By the Schwarz reflection principle, the $f_{t}$ extend analytically across $\mathbb{T} \backslash A$ via the formula $f_{t}(z)=-\overline{f_{t}(1 / \bar{z})}$ which even extends analytically to $\infty$. The singularities at the $a_{j}$ are either removable or simple poles. (Without going into too much detail we can give an elementary explanation. The derivative of $f_{t}$ cannot vanish on $\mathbb{T} \backslash A$ because of local mapping properties. Thus, $f_{t}\left(e^{i \theta}\right)$ is imaginary-valued and monotone off singularities and this is enough to prove $f_{t}$ omits an imaginary line segment near singularities. This implies $f_{t}$ cannot have an essential singularity at any $a_{j}$ by either the Big Picard theorem or by conformal mapping and the Casorati-Weierstrass theorem. Any poles on $\mathbb{T}$ must be simple because of local mapping properties of $1 / f_{t}$.) Therefore, the $f_{t}$ are rational and by their symmetry properties we get

$$
\begin{aligned}
f_{1}(z) & =\frac{p(z)+\tilde{p}(z)}{p(z)-\tilde{p}(z)}+\frac{v(z)}{\frac{\overline{p(z)-\tilde{p}(z)}}{\overline{p(1 / \bar{z})}+\overline{\tilde{p}(1 / \bar{z})}}}-\frac{\overline{v(1 / \bar{z})}}{\overline{\overline{p(1 / \bar{z})}-\overline{\tilde{p}(1 / \bar{z})}}} \\
& \left.=-\overline{f_{1}(1 / \bar{z})}=-\overline{\tilde{p}(1 / \bar{z})}-\bar{z}\right)
\end{aligned}
$$


and after some simplification we get

$$
\frac{v(z)}{p(z)-\tilde{p}(z)}=\frac{z^{n} \overline{v(1 / \bar{z})}}{p(z)-\tilde{p}(z)}
$$

for $z \notin A$. Necessarily, $v$ has no poles (because simple poles in the above function are accounted for by the denominator) and hence $v$ must be a polynomial. Also, $v(z)=z^{n} \overline{v(1 / \bar{z})}$ and $v(0)=0$.

Thus, every element of the face associated to $f$ is of the form

$$
\frac{p+\tilde{p}}{p-\tilde{p}}+\frac{2 v}{p-\tilde{p}}
$$

where $v$ is a polynomial such that $v=\tilde{v}, v(0)=0, p+v$ has no zeros in $\mathbb{D}$, and there exists $a>0$ such that $p-a v$ has no zeros in $\mathbb{D}$.

Conversely, given $v$ satisfying all of the above conditions, $\frac{\tilde{p}+v}{p+v}, \frac{\tilde{p}-a v}{p-a v}$ are finite Blaschke products, and $f=\frac{p+\tilde{p}}{p-\tilde{p}}$ is a convex combination of $f_{1}, f_{-a}$ as defined above.

This gives the desired characterization of faces in the one variable case.

Next we look at several variables. Write $f=\frac{p+\tilde{p}}{p-\tilde{p}} \in P R P R_{d}$ where $p \in \mathbb{C}\left[z_{1}, \ldots, z_{d}\right]$ is scattering stable and has multidegree at most $n=\left(n_{1}, \ldots, n_{d}\right)$.

Now, if $f+t H \in P R P_{d}$ for $t \in[-a, 1]$ and $H=\frac{2 v}{p-\tilde{p}}$ for some analytic $v: \mathbb{D}^{d} \rightarrow \mathbb{C}$, then we claim $v$ is a polynomial of degree at most $n$ with $v=\tilde{v}$.

We examine slices $f_{\zeta}(z):=f(z \zeta)$ where $z \in \mathbb{D}, \zeta \in \mathbb{T}^{d}$. We will use this notation for other functions, not just $f$.

If we verify the hypotheses of the following lemma, then we are finished. The lemma will be proven at the end.

Lemma 2.1. Let $h: \mathbb{D}^{d} \rightarrow \mathbb{C}$ be analytic. If for each $\zeta \in \mathbb{T}^{d}, h_{\zeta}(z)$ is a (one variable) polynomial of degree at most $|n|=n_{1}+\cdots+n_{d}$ satisfying $h_{\zeta}(z)=\zeta^{n} z^{|n|} \overline{h_{\zeta}(1 / \bar{z})}$, then $h$ is a (d variable) polynomial of multidegree at most $n$ and $h=\tilde{h}$.

Fix $\zeta \in \mathbb{T}^{d}$. Since $p$ has multidegree at most $n, p_{\zeta}$ has degree at most $|n|$ and we can calculate that

Then, for a choice of $\mu=\sqrt{\zeta^{n}}$

$$
\zeta^{n} \widetilde{p}_{\zeta}(z)=\tilde{p}_{\zeta}(z)
$$

$$
f_{\zeta}=\frac{p_{\zeta}+\zeta^{n} \widetilde{p_{\zeta}}}{p_{\zeta}-\zeta^{n} \widetilde{p_{\zeta}}}=\frac{\bar{\mu} p_{\zeta}+\mu \widetilde{p_{\zeta}}}{\bar{\mu} p_{\zeta}-\mu \widetilde{p_{\zeta}}}
$$

The point is that we are matching the earlier formulation of the Cayley transform. However, in this case it is possible for $p_{\zeta}$ and $\widetilde{p}_{\zeta}$ to have common zeros, necessarily on $\mathbb{T}$. Let $q$ be a greatest common divisor of $p_{\zeta}$ and $\widetilde{p}_{\zeta}$. We can arrange for $q=\tilde{q}$ by multiplying by an appropriate constant since all of the roots of $q$ will be on $\mathbb{T}$. Let $g=\bar{\mu} p_{\zeta} / q$ which has degree at most $|n|-\operatorname{deg} q$.

Then, $f_{\zeta}=\frac{g+\tilde{g}}{g-\tilde{g}}$ and $H_{\zeta}=\frac{2 \bar{\mu} v_{\zeta} / q}{g-\tilde{g}}$. We can now apply the one variable result to see that $\bar{\mu} v_{\zeta} / q$ is a polynomial of degree $|n|-\operatorname{deg} q$ and is equal to its reflection which means

$$
\bar{\mu} v_{\zeta}(z) / q(z)=\mu z^{|n|} \overline{v_{\zeta}(1 / \bar{z})} / q(z)
$$

and this implies the hypotheses of Lemma 2.1, proven below. This lemma can essentially by found in McDonald [12 but we include a proof for convenience. 
Proof of Lemma 2.1. We write out the power series $h(w)=\sum_{\alpha} a_{\alpha} w^{\alpha}$, so that

$$
\begin{aligned}
h_{\zeta}(z) & =\sum_{\alpha} a_{\alpha} z^{|\alpha|} \zeta^{\alpha} \\
& =\sum_{j \geq 0} z^{j}\left(\sum_{\alpha:|\alpha|=j} a_{\alpha} \zeta^{\alpha}\right)
\end{aligned}
$$

and we see that for $j>|n|$, we have $0 \equiv \sum_{|\alpha|=j} a_{\alpha} \zeta^{\alpha}$ and therefore $a_{\alpha}=0$ for $|\alpha|>|n|$ since this is an identically zero trigonometric polynomial.

The reflection condition on $h_{\zeta}$ implies for $0 \leq j \leq|n|$

$$
\sum_{\alpha:|\alpha|=j} a_{\alpha} \zeta^{\alpha}=\sum_{\alpha:|\alpha|=|n|-j} \overline{a_{\alpha}} \zeta^{n-\alpha} .
$$

By matching Fourier coefficients, we see that $a_{\alpha}=0$ if for some $k$ we have $\alpha_{k}>n_{k}$. Thus, $h$ has multidegree at most $n$ and the sum on the right can be reindexed as

$$
\sum_{\alpha:|\alpha|=j} \overline{a_{n-\alpha}} \zeta^{\alpha}
$$

(where $a_{\beta}=0$ if undefined) so that $a_{\alpha}=\overline{a_{n-\alpha}}$. This exactly means $h=\tilde{h}$ if we examine coefficients.

This proves McDonald's characterization of faces with the additional information that $v_{\zeta}$ vanishes to at least the same order at a point of $\mathbb{T}$ as $p_{\zeta}$. Using work of [10] it is possible to show $v$ vanishes to at least the same order as $p$ at a point of $\mathbb{T}^{d}$.

Proof of Theorem 1.3. We may assume $\zeta=1:=(1, \ldots, 1)$. Theorem 14.1 of [10] says that if $p \in \mathbb{C}\left[z_{1}, \ldots, z_{d}\right]$ has no zeros in $\mathbb{D}^{d}$ and $p$ vanishes at $\mathbf{1}$ to order $M$, meaning

$$
p(\mathbf{1}-\zeta)=\sum_{j=M}^{N} P_{M}(\zeta)
$$

where the $P_{j}$ are homogeneous of total degree $j$ and $P_{M} \neq 0$, then $P_{M}$ has no zeros in $R H P^{d}$. Then, the order of vanishing of $p_{\mathbf{1}}(z)=p(z \mathbf{1})$ at $z=1$ equals $M$ because $P_{M}((1-z) \mathbf{1})=$ $(1-z)^{M} P_{M}(\mathbf{1})$ and $P_{M}(\mathbf{1}) \neq 0$. (Geometrically, the variety $p=0$ has no radial tangents on $\left.\mathbb{T}^{d}.\right)$

Note both $p$ and $p+v$ have no zeros in $\mathbb{D}^{d}$. Thus, if $p$ vanishes to order $M$ at $\mathbf{1}$, then $p_{\mathbf{1}}$ vanishes to order $M$ at 1 . In turn, $v_{\mathbf{1}}$ vanishes to at least order $M$, and then so does $(p+v)_{\mathbf{1}}$, and we then conclude the order of vanishing of $p+v$ at $\mathbf{1}$ is at least $M$.

If we use an additional result from [10] we can say something about the bottom homogeneous term of $v$ at 1 .

Proposition 14.5 of [10] says that if $p \in \mathbb{C}\left[z_{1}, \ldots, z_{d}\right]$ has no zeros in $\mathbb{D}^{d}$ and vanishes to order $M$ at 1 then writing homogeneous expansions:

$$
p(\mathbf{1}-\zeta)=\sum_{j=M}^{N} P_{j}(\zeta) \quad \tilde{p}(\mathbf{1}-\zeta)=\sum_{j=M}^{N} Q_{j}(\zeta)
$$

we have that $Q_{M}$ is a unimodular multiple of $P_{M}$, say $Q_{M}=\mu^{2} P_{M}$ for some $\mu \in \mathbb{T}$. (It will be convenient to take a square root of $\mu^{2}$ later.) Set $F=\tilde{p} / p$. It is not hard to prove 
$F(\mathbf{1}-\zeta) \rightarrow \mu^{2}$ as $\zeta \rightarrow 0$ non-tangentially in $R H P^{d}$. The details are in Proposition 14.3 of [10].

We also need to write out the homogeneous expansion of $h$

$$
v(\mathbf{1}-\zeta)=\sum_{j=M}^{N} V_{j}(\zeta)
$$

By the above work, $v$ vanishes at least to order $M$ at $\mathbf{1}$. It is possible that $v$ vanishes to higher order, so we allow for $V_{M}=0$.

We assume for simplicity that $p \pm v$ have no zeros in $\mathbb{D}^{d}$; this is true after rescaling $v$. The following inequalities hold

$$
|p \pm v|^{2}-|\tilde{p} \pm v|^{2} \geq 0 \text { on } \mathbb{D}^{d}
$$

(see Lemma 14.4 of [10]). We can rewrite this as

$$
|p(z)|^{2}-|\tilde{p}(z)|^{2} \geq 2|\operatorname{Re}((\overline{p(z)}-\overline{\tilde{p}(z)}) v(z))| .
$$

Let $\zeta \in R H P^{d}$ and set $z=z(t)=\mathbf{1}-t \zeta$. For $t>0$ small enough $z(t) \in \mathbb{D}^{d}$ and since $P_{M}$ is a unimodular multiple of $Q_{M},|p(z(t))|^{2}-|\tilde{p}(z(t))|^{2}$ vanishes to order at least $M(M+1)$ in $t$. This must also hold for the right hand side of (2.1) which implies

$$
\operatorname{Re}\left(\left(1-\bar{\mu}^{2}\right) \overline{P_{M}(\zeta)} V_{M}(\zeta)\right)=0
$$

which implies $\left(1-\bar{\mu}^{2}\right) \frac{V_{M}}{P_{M}}=i c$ for some $c \in \mathbb{R}$.

If $\mu^{2} \neq 1$, then the constant $\frac{i c}{1-\bar{\mu}^{2}}$ is of the form $r \mu$ for $r \in \mathbb{R}$. We summarize the above.

Theorem 2.2. Assume the setup of Theorem 1.2. Suppose p vanishes at 1 to order $M$. Let $\mu^{2}=F(\mathbf{1})$ in the sense of a non-tangential limit. Assume $\mu^{2} \neq 1$. Then the bottom homogeneous terms of $p(\mathbf{1}-\zeta), v(\mathbf{1}-\zeta)$ at $\zeta=0$, say $P_{M}, V_{M}$, satisfy

$$
V_{M}=r \mu P_{M}
$$

for some $r \in \mathbb{R}$.

This result is strong enough to prove $g_{2}$ from the introduction is extreme. Note that for $p\left(z_{1}, z_{2}\right)=2-z_{1}-z_{2}$ we have

$$
\begin{gathered}
p\left(1-\zeta_{1}, 1-\zeta_{2}\right)=\zeta_{1}+\zeta_{2} \\
\tilde{p}\left(1-\zeta_{1}, 1-\zeta_{2}\right)=-\zeta_{1}-\zeta_{2}+2 \zeta_{1} \zeta_{2}
\end{gathered}
$$

which means $\mu^{2}=-1$. Then, any perturbation of $p$ must be of the form $v\left(z_{1}, z_{2}\right)=\operatorname{ir} z_{1}-i r z_{2}$ where $r \in \mathbb{R}$ and

$$
v\left(1-\zeta_{1}, 1-\zeta_{2}\right)=i r\left(\zeta_{2}-\zeta_{1}\right)
$$

which is not a multiple of $\zeta_{1}+\zeta_{2}$ unless $r=0$.

The theorem says nothing when $\mu^{2}=1$ and this is for good reason. Consider

$$
q=i\left(2-z_{1}-z_{2}\right)
$$

For $G=\frac{\tilde{q}}{q}$ we have $\lim _{r} \nearrow_{1} G(r, r)=1$ and $g=(1+G) /(1-G)$ is not extreme:

$$
g=\frac{1-z_{1} z_{2}}{\left(1-z_{1}\right)\left(1-z_{2}\right)}=\frac{1}{2}\left(\frac{1+z_{1}}{1-z_{1}}+\frac{1+z_{2}}{1-z_{2}}\right) .
$$




\section{Preliminaries for Theorems 1.5 and 1.6}

McDonald's theorem reduces the possibilities for the face of a given $f$. If $f$ is built out of a $\mathbb{T}^{2}$-saturated polynomial, we can limit $f$ 's face further.

First, let us recall the Schur-Cohn test.

Theorem 3.1 (Schur-Cohn test). Let $p \in \mathbb{C}[z]$ and write $p(z)=\sum_{j=0}^{m} p_{j} z^{j}$. Define

$$
P=\left(\begin{array}{cccc}
p_{0} & p_{1} & \ldots & p_{m-1} \\
0 & p_{0} & \ldots & p_{m-2} \\
\vdots & 0 & \ddots & \vdots \\
0 & 0 & 0 & p_{0}
\end{array}\right) \quad Q=\left(\begin{array}{cccc}
\overline{p_{m}} & \overline{p_{m-1}} & \cdots & \overline{p_{1}} \\
0 & \overline{p_{m}} & \cdots & \overline{p_{2}} \\
\vdots & 0 & \ddots & \vdots \\
0 & 0 & 0 & \overline{p_{m}}
\end{array}\right)
$$

Then, $p$ has no zeros in $\overline{\mathbb{D}}$ if and only if $P^{*} P-Q^{*} Q>0$.

Actually this result is due to Schur while Cohn proved a generalization (see [14]).

We can apply this to bivariate polynomials $p \in \mathbb{C}[z, w]$ of bidegree $(n, m)$ as follows. Write $p(z, w)=\sum_{j=0}^{m} p_{j}(z) w^{j}$. Then, $\tilde{p}(z, w)=\sum_{j=0}^{m} \tilde{p}_{m-j}(z) w^{j}$ where $\tilde{p}_{j}(z)=z^{n} \overline{p_{j}(1 / \bar{z})}$. Note that we use $(z, w)$ for the coordinates in $\mathbb{C}^{2}$ instead of $\left(z_{1}, z_{2}\right)$.

Define

$$
P(z)=\left(\begin{array}{cccc}
p_{0}(z) & p_{1}(z) & \ldots & p_{m-1}(z) \\
0 & p_{0}(z) & \ldots & p_{m-2}(z) \\
\vdots & 0 & \ddots & \vdots \\
0 & 0 & 0 & p_{0}(z)
\end{array}\right) \quad Q(z)=\left(\begin{array}{cccc}
\tilde{p}_{m}(z) & \tilde{p}_{m-1}(z) & \ldots & \tilde{p}_{1}(z) \\
0 & \tilde{p}_{m}(z) & \ldots & \tilde{p}_{2}(z) \\
\vdots & 0 & \ddots & \vdots \\
0 & 0 & 0 & \tilde{p}_{m}(z)
\end{array}\right)
$$

Note that for $z \in \mathbb{T}, Q(z)$ is almost a direct analogue of $Q$ above; it differs by a factor of $z^{n}$. This gets cancelled out in the Schur-Cohn matrix calculation.

Indeed, $p$ has no zeros in $\mathbb{T} \times \overline{\mathbb{D}}$ if and only if $T_{p}(z)=P(z)^{*} P(z)-Q(z)^{*} Q(z)>0$ for all $z \in \mathbb{T}$.

The following formula holds for $z \in \mathbb{T}, w, \eta \in \mathbb{C}$

$$
\frac{\overline{p(z, \eta)} p(z, w)-\overline{\tilde{p}(z, \eta)} \tilde{p}(z, w)}{1-\bar{\eta} w}=\left(1, \bar{\eta}, \ldots, \bar{\eta}^{m-1}\right) T_{p}(z)\left(1, w, \ldots, w^{m-1}\right)^{t} .
$$

Lemma 3.2. If $p$ has no zeros in $\mathbb{T} \times \mathbb{D}$ or no zeros in $\mathbb{D}^{2}$, then $P(z)^{*} P(z)-Q(z)^{*} Q(z) \geq 0$ for all $z \in \mathbb{T}$.

Proof. If $p$ has no zeros in $\mathbb{T} \times \mathbb{D}$, then the polynomial $p_{r}(z, w)=p(z, r w)$ has no zeros in $\mathbb{T} \times \overline{\mathbb{D}}$ for $r \in(0,1)$. We can then form analogues of $P, Q$ corresponding to $p_{r}, \tilde{p}_{r}$ which we label $P_{r}, Q_{r}$. The dependence on $r$ is evidently continuous. Then, $P_{r}^{*} P_{r}-Q_{r}^{*} Q_{r}>0$ on $\mathbb{T}$. If we send $r \nearrow 1$, then $P^{*} P-Q^{*} Q \geq 0$ by continuity.

If $p$ has no zeros in $\mathbb{D}^{2}$, then $p(r z, w)$ has no zeros in $\mathbb{T} \times \mathbb{D}$ for $r \in(0,1)$. We can again form analogues, say $P^{r}, Q^{r}$, of $P, Q$ depending on $r$ for which $\left(P^{r}\right)^{*} P^{r}-\left(Q^{r}\right)^{*} Q^{r} \geq 0$ on $\mathbb{T}$. Then, send $r \nearrow 1$ to see $P^{*} P-Q^{*} Q \geq 0$ on $\mathbb{T}$.

Lemma 3.3. If $p$ is scattering stable, then $p$ has no zeros in $(\mathbb{T} \times \mathbb{D}) \cup(\mathbb{D} \times \mathbb{T})$.

Proof. For fixed $z_{0} \in \mathbb{D}, p\left(z_{0}, \cdot\right)$ has no zeros in $\mathbb{D}$. By Hurwitz's theorem, if we send $z_{0}$ to $\mathbb{T}$, we see that $p\left(z_{0}, \cdot\right)$ either has no zeros in $\mathbb{D}$ or is identically zero. In the latter case, $z-z_{0}$ divides $p$ and hence also $\tilde{p}$ since $z_{0} \in \mathbb{T}$. Thus, $p$ is non-vanishing on $\mathbb{T} \times \mathbb{D}$ and by symmetry on $\mathbb{D} \times \mathbb{T}$. 
Lemma 3.4. Let $p \in \mathbb{C}[z, w]$ and form $P(z), Q(z)$ as above. Then, $P(z)^{*} P(z)-Q(z)^{*} Q(z)>$ 0 for all but finitely many $z \in \mathbb{T}$ if and only if $p$ has no zeros in $(\mathbb{T} \times \overline{\mathbb{D}}) \backslash S$ where $S$ consists of finitely many points in $\mathbb{T}^{2}$ and finitely many "vertical" lines $z=z_{0}$ with $z_{0} \in \mathbb{T}$.

We can rule out vertical lines if we assume $p$ and $\tilde{p}$ have no common factors.

We note that the condition $P^{*} P-Q^{*} Q>0$ for all but finitely many $z \in \mathbb{T}$ is equivalent to saying $P^{*} P-Q^{*} Q \geq 0$ and $\operatorname{det}\left(P^{*} P-Q^{*} Q\right)$ is not identically zero.

Proof. By the Schur-Cohn test, $P(z)^{*} P(z)-Q(z)^{*} Q(z)>0$ for all but finitely many $z \in \mathbb{T}$ if and only if $w \mapsto p(z, w)$ has no zeros in $\overline{\mathbb{D}}$ for all but finitely many $z \in \mathbb{T}$. The latter condition means there exists a finite set $S_{1} \subset \mathbb{T}$ such that $w \mapsto p(z, w)$ has no zeros in $\overline{\mathbb{D}}$ except for when $z \in S_{1}$. By Hurwitz's theorem, if $z_{0} \in S_{1}$ then $w \mapsto p\left(z_{0}, w\right)$ either has no zeros in $\mathbb{D}$ or is identically zero. In the latter case, $z-z_{0}$ divides $p$ which means $p$ vanishes on a vertical line. In the former case, $p\left(z_{0}, \cdot\right)$ has no zeros in $\mathbb{D}$ (although it could have zeros in $\mathbb{T}$ ). Thus, the condition $P^{*} P-Q^{*} Q>0$ for all but finitely many $z$ is equivalent to $p$ having no zeros in $(\mathbb{T} \times \overline{\mathbb{D}}) \backslash S$ where $S$ consists of finitely many vertical lines and finitely many points of $\mathbb{T}^{2}$.

Lemma 3.5. Assume $p \in \mathbb{C}[z, w]$ has bidegree $(n, m)$. Let $r(z)$ be the resultant of $p$ and $\tilde{p}$ with respect to the variable $w$. Then, on $\mathbb{T}$

$$
r(z)=z^{n m} \operatorname{det}\left(P(z)^{*} P(z)-Q(z)^{*} Q(z)\right)
$$

where $P, Q$ are defined in (3.1). In particular, the polynomials $p$ and $\tilde{p}$ have a common factor involving $w$ if and only if $\operatorname{det}\left(P(z)^{*} P(z)-Q(z)^{*} Q(z)\right)=0$ for all $z \in \mathbb{T}$.

Proof. This proof is from [6] (specifically Lemma 2.1.3 of that paper, which says their proof is inspired by similar arguments for Bezoutians in [11], Theorem 1 Section 13.3).

Let $\tilde{P}(z)=z^{n} P(1 / \bar{z})^{*}$ and $\tilde{Q}(z)=z^{n} Q(1 / \bar{z})^{*}$. Then,

$$
\begin{aligned}
R(z)= & \left(\begin{array}{ccccc}
P(z) & \tilde{Q}(z) \\
Q(z) & \tilde{P}(z)
\end{array}\right) \\
= & \left(\begin{array}{cccccc}
p_{0}(z) & \cdots & p_{m-1}(z) & p_{m}(z) & & \bigcirc \\
& \ddots & \vdots & \vdots & \ddots & \\
\bigcirc & & p_{0}(z) & p_{1}(z) & \cdots & p_{m}(z) \\
\tilde{p}_{m}(z) & \cdots & \tilde{p}_{1}(z) & \tilde{p}_{0}(z) & & \bigcirc \\
& \ddots & \vdots & \vdots & \ddots & \\
\bigcirc & & \tilde{p}_{m}(z) & \tilde{p}_{m-1}(z) & \cdots & \tilde{p}_{0}(z)
\end{array}\right)
\end{aligned}
$$

is the resultant or Sylvester matrix of $p$ and $\tilde{p}$ with respect to $w$ and its determinant is the resultant $r$ of $p$ and $\tilde{p}$ with respect to $w$. Since $P$ and $Q$ commute, we can compute

$$
r(z)=\operatorname{det}(\tilde{P}(z) P(z)-\tilde{Q}(z) Q(z))
$$

and on $\mathbb{T}$ this agrees with $z^{n m} \operatorname{det}\left(P(z)^{*} P(z)-Q(z)^{*} Q(z)\right)$. Since $\mathbb{T}$ is a set of uniqueness, this determinant is identically zero on $\mathbb{T}$ if and only if the resultant $r \equiv 0$ which by standard properties of the resultant holds if and only if $p$ and $\tilde{p}$ have a common factor depending on $w$. 
We shall begin the proofs of Theorem 1.5 and Theorem[1.6 simultaneously and then diverge at a certain point.

Let $f=\frac{p+\tilde{p}}{p-\tilde{p}}$ be in $P R P R_{2}$ with associated scattering stable polynomial $p \in \mathbb{C}[z, w]$.

By McDonald's characterization, points of the faces of $f$ are associated to $v \in \mathbb{C}[z, w]$ satisfying: $v(0)=0, \operatorname{deg} v=\operatorname{deg} \tilde{p}, v=\tilde{v}$, and there is an interval $I=[a, b]$ with $a<0<b$ such that for $t \in I, p+t v$ has no zeros in $\mathbb{D}^{2}$. For our purposes, we can shrink the interval $I$ to be symmetric about 0 and rescale $v$ so that $p \pm v$ have no zeros in $\mathbb{D}^{2}$ and in fact $p \pm v$ are scattering stable. This is because the resultants associated to $p, \tilde{p}$ will not be identically zero and thus so will those of $p+t v, \tilde{p}+t v$ for $|t|$ small.

Write $v=\sum_{j=0}^{m} v_{j}(z) w^{j}$ and form

$$
V(z)=\left(\begin{array}{cccc}
v_{0}(z) & v_{1}(z) & \ldots & v_{m-1}(z) \\
0 & v_{0}(z) & \ldots & v_{m-2}(z) \\
\vdots & 0 & \ddots & \vdots \\
0 & 0 & 0 & v_{0}(z)
\end{array}\right) .
$$

All of the matrix functions in the rest of this proof will be functions of $z$, so we will omit the evaluations such as $V(z)$ and simply write $V$. Since $p \pm v$ are scattering stable, $(P \pm V)^{*}(P \pm V)-(Q \pm V)^{*}(Q \pm V)>0$ for all but finitely many $z \in \mathbb{T}$.

By the matrix Fejér-Riesz lemma, we can factor

$$
(P \pm V)^{*}(P \pm V)-(Q \pm V)^{*}(Q \pm V)=E_{ \pm}^{*} E_{ \pm}
$$

where $E_{+}, E_{-}$are matrix polynomials of degree at most $n$ with $\operatorname{det} E_{ \pm}(z)$ non-zero for $z \in \mathbb{D}$. We can also factor

$$
P^{*} P-Q^{*} Q=E^{*} E
$$

where $E$ is a matrix polynomial of degree at most $n$ and is non-singular whenever $z \in \mathbb{D}$.

Let $r(z)$ be the resultant of $p$ and $\tilde{p}$ with respect to $w$, and let $r_{ \pm}(z)$ be the resultants of $p \pm v, \tilde{p} \pm v$ with respect to $w$ (for the choices of + and - ). The number of roots of the resultant $r(z)$ on $\mathbb{T}$ equals the number of common roots of $p$ and $\tilde{p}$ on $\mathbb{T}^{2}$ since $p$ has no zeros on $\mathbb{D} \times \mathbb{T}$ by Lemma 3.3. More precisely, the multiplicity of a given root of $r(z)$, say $z_{0}$, counts the number of common roots of $p$ and $\tilde{p}$ on the line $z=z_{0}$. Note that $r(z)=z^{m n}|\operatorname{det}(E)|^{2}$ and $r_{ \pm}(z)=z^{m n}\left|\operatorname{det}\left(E_{ \pm}\right)\right|^{2}$ on $\mathbb{T}$.

The left side of (3.3) is

$$
P^{*} P-Q^{*} Q-( \pm 1) 2 \operatorname{Re}\left((P-Q)^{*} V\right)
$$

and averaging over + and - yields

$$
P^{*} P-Q^{*} Q=E^{*} E=\frac{1}{2}\left(E_{+}^{*} E_{+}+E_{-}^{*} E_{-}\right)
$$

on $\mathbb{T}$. Setting $\Phi_{ \pm}=E_{ \pm} E^{-1}$ we have $2 I=\Phi_{+}^{*} \Phi_{+}+\Phi_{-}^{*} \Phi_{-}$on $\mathbb{T}$ away from poles of $E^{-1}$. But, this equation shows $\Phi_{ \pm}$are bounded on $\mathbb{T}$ and so cannot have any poles. So, $\Phi_{ \pm}$are analytic on $\overline{\mathbb{D}}$ since we already know $E^{-1}$ has no poles in $\mathbb{D}$.

We conclude that on $\mathbb{T}, r_{ \pm}(z)=r(z)\left|\operatorname{det}\left(\Phi_{ \pm}\right)\right|^{2}$. Let $I_{\left\{z=z_{0}\right\}}(p, q)$ denote the number of common zeros of two polynomials on the line $z=z_{0}$ counting multiplicities. We have just proved the following. 
Proposition 3.6. Suppose $p \in \mathbb{C}[z, w]$ is scattering stable, and suppose $v \in \mathbb{C}[z, w]$ satisfies $v(0)=0, \operatorname{deg} v=\operatorname{deg} \tilde{p}, v=\tilde{v}$, as well as $p \pm v$ are scattering stable. Then, for $z_{0} \in \mathbb{T}$

$$
I_{\left\{z=z_{0}\right\}}(p, \tilde{p}) \leq I_{\left\{z=z_{0}\right\}}(p \pm v, \tilde{p} \pm v) .
$$

Moreover, both intersection multiplicities are even.

The last statement follows from the formula $r(z)=z^{m n}|\operatorname{det} E|^{2}$ which implies that zeros on $\mathbb{T}$ occur with even multiplicity.

If the common zeros of $p$ and $\tilde{p}$ on $\mathbb{T}^{2}$ had distinct $z$-coordinates (or $w$-coordinates) then we would be finished with Theorem 1.6.

At this stage the reader can jump to the next section to finish the proof of Theorem 1.5 or Section 5 to finish the proof of Theorem 1.6 .

\section{Completion of the proof of Theorem 1.5}

Assume now that $p$ is $\mathbb{T}^{2}$-saturated and $p-\tilde{p}$ is irreducible. Then, the resultant of $p$ and $\tilde{p}, r(z)$, has $2 n m$ roots on $\mathbb{T}$ and these must be all of its roots since $r(z)$ has degree at most $2 n m$. This implies $\operatorname{det}\left(P^{*} P-Q^{*} Q\right)=\bar{z}^{n m} r(z)$ has $2 n m$ roots on $\mathbb{T}$ and therefore det $E$ has $n m$ roots on $\mathbb{T}$. These must be all of the roots of $\operatorname{det} E$ since $E$ is $m \times m$ and of degree at most $n$. Thus, $E$ is invertible in $\mathbb{D}$ and $\mathbb{C} \backslash \overline{\mathbb{D}}$. Also, $z^{n} E(1 / z)$ is an $m \times m$ matrix polynomial of degree at most $n$, which implies $\operatorname{det}\left(z^{n} E(1 / z)\right)$ has degree at most $n m$. Since det $E$ has $n m$ roots on $\mathbb{T}$, we can conclude that $\operatorname{det}\left(z^{n} E(1 / z)\right)$ has all of its roots on $\mathbb{T}$. Thus, $z^{n} E(1 / z)$ is invertible at 0 .

At the same time,

$$
E_{ \pm}(1 / z) E^{-1}(1 / z)=\left(z^{n} E_{ \pm}(1 / z)\right)\left(z^{n} E(1 / z)\right)^{-1}
$$

is analytic on $\mathbb{D}$ since $z^{n} E(1 / z)$ is invertible in $\mathbb{D}$. This implies $\Phi_{ \pm}$are analytic on the Riemann sphere, which implies $\Phi_{ \pm}$are constant $m \times m$ matrices.

Subtract equation (3.3) with + from - to obtain

$$
4 \operatorname{Re}\left((P-Q)^{*} V\right)=E_{-}^{*} E_{-}-E_{+}^{*} E_{+}=E^{*}\left(\Phi_{-}^{*} \Phi_{-}-\Phi_{+}^{*} \Phi_{+}\right) E .
$$

Now we consider the matrices obtained from $p+t v$ where $t$ is a real parameter. The matrices we get are

$$
(P+t V)^{*}(P+t V)-(Q+t V)^{*}(Q+t V)=E^{*}\left(I-t \frac{1}{2} \Phi_{-}^{*} \Phi_{-}+t \frac{1}{2} \Phi_{+}^{*} \Phi_{+}\right) E .
$$

Unless $\Phi_{-}^{*} \Phi_{-}=\Phi_{+}^{*} \Phi_{+}$, there will be a value of $t$ such that

$$
\left(I-t\left(\frac{1}{2} \Phi_{-}^{*} \Phi_{-}-\frac{1}{2} \Phi_{+}^{*} \Phi_{+}\right)\right)
$$

is singular since all matrices involved are self-adjoint. For such $t$, $\operatorname{det}\left((P+t V)^{*}(P+t V)-\right.$ $\left.(Q+t V)^{*}(Q+t V)\right)$ is identically zero on $\mathbb{T}$ and by Lemma 3.5 this implies $p+t v$ and $\tilde{p}+t v$ have a common factor. Such a common factor must be proper (meaning it has strictly lower degree in one of the variables).

Indeed, if it is not proper, then $p+t v$ divides $\tilde{p}+t v$ or vice versa. The latter possibility is excluded by the fact $p(0) \neq 0=\tilde{p}(0)=v(0)$. In the former case, $\tilde{p}+t v=(p+t v) g$ for some non-constant polynomial $g$ with $g(0)=0$. (Note we could not immediately rule this case out based on degrees because $p+t v$ only has degree at most $(n, m)$.) Then, $p-\tilde{p}=(p+t v)(1-g)$ 
is reducible contrary to our assumption. However, a proper common factor of $p+t v$ and $\tilde{p}+t v$ implies a proper factor of $p-\tilde{p}$, which again contradicts irreducibility of $p-\tilde{p}$.

We are left with the possibility $\Phi_{-}^{*} \Phi_{-}=\Phi_{+}^{*} \Phi_{+}$. By (4.1) we have $\operatorname{Re}\left((P-Q)^{*} V\right) \equiv 0$, which implies

$$
(P+t V)^{*}(P+t V)-(Q+t V)^{*}(Q+t V)=P^{*} P-Q^{*} Q
$$

for all $t \in \mathbb{R}$. By (3.2), this implies for $z \in \mathbb{T}, w, \eta \in \mathbb{C}$ that the following expression does not depend on $t$

$$
\frac{\overline{(p+t v)(z, \eta)}(p+t v)(z, w)-\overline{(\tilde{p}+t v)(z, \eta)}(\tilde{p}+t v)(z, w)}{1-\bar{\eta} w} .
$$

The coefficient of $t$ is therefore identically zero yielding

$$
\overline{(p-\tilde{p})(z, \eta)} v(z, w)+\overline{v(z, \eta)}(p-\tilde{p})(z, w)=0
$$

for $w, \eta \in \mathbb{C}, z \in \mathbb{T}$. Since $z \in \mathbb{T}$ and $v=\tilde{v}$, if we replace $\eta$ with $1 / \bar{\eta}$ and multiply through by $z^{n} \eta^{m}$ we get

$$
(\tilde{p}-p)(z, \eta) v(z, w)+v(z, \eta)(p-\tilde{p})(z, w)=0
$$

for $w, \eta \in \mathbb{C}$. As this is a polynomial in $z$ the identity holds for all $z \in \mathbb{C}$, not just $\mathbb{T}$. Since $p-\tilde{p}$ is assumed to be irreducible, either:

(1) $(p-\tilde{p})(z, w)$ divides $(\tilde{p}-p)(z, \eta)$ or

(2) $(p-\tilde{p})(z, w)$ divides $v(z, w)$.

In addressing these two cases it is useful to point out that $p-\tilde{p}$ has bidegree exactly $(n, m)$. This is because $p(0) \neq 0$ and the reflection of $p-\tilde{p}$ at the degree $(n, m)$ is $\tilde{p}-p$ which implies the coefficient of $z^{n} w^{m}$ in $(p-\tilde{p})(z, w)$ is $-\overline{p(0)}$.

Case (1) implies $(p-\tilde{p})(z, w)$ does not depend on $w$ which is only possible if $m=0$. But,

$p-\tilde{p}$ being irreducible means $n=0$ or $n=1$ by the fundamental theorem of algebra. Having $n=0$ is not allowed and when $n=1$ we automatically have $v=0$.

Case (2) implies $v$ is a multiple of $p-\tilde{p}$ since $v$ has degree at most $(n, m)$ and $p-\tilde{p}$ has degree exactly $(n, m)$. Since $p(0) \neq 0$ this is impossible unless $v=0$.

In all cases $v=0$. This means the face of $f$ is the singleton $\{f\}$ and proves $f$ is an extreme point.

\section{Completion of the proof of Theorem 1.6}

We pick up where we left off in Section 3. Proposition 3.6 shows the number of common zeros of $p$ and $\tilde{p}$ on $\mathbb{T}^{2}$ is less than or equal to the number of common zeros of $p \pm v$ and $\tilde{p} \pm v$ on $\mathbb{T}^{2}$. However, since the resultant is a global object we have only proven that the number of zeros on "vertical" lines in $\mathbb{T}^{2}$ increases. To fix this we cannot do a linear change of variables as is often done when using the resultant to count common zeros (as in [3] for instance). Instead we perform a more complicated change of variables and use a different (but equivalent) definition of intersection multiplicity.

First, recall that the intersection multiplicity of $p, q$ at a common zero $x \in \mathbb{C}^{2}$ is equal to

$$
\operatorname{dim} \mathcal{O}_{x} /(p, q) \mathcal{O}_{x}
$$

Here $\mathcal{O}_{x}$ is the localization of $\mathbb{C}[z, w]$ at $x$, namely the ring of rational functions with denominators non-vanishing at $x$. Also, $(p, q) \mathcal{O}_{x}$ denotes the ideal generated by $p, q$ within the local ring $\mathcal{O}_{x}$. This definition of intersection multiplicity is used in [4]. 
Now, suppose $p, \tilde{p}$ have a common zero on $\mathbb{T}^{2}$ which we assume without loss of generality is $\mathbf{1}=(1,1)$. The map

$$
\phi_{k}: F(z, w) \mapsto F\left(w^{k} z, w\right)
$$

is an isomorphism of the local ring $\mathcal{O}_{\mathbf{1}}$ onto itself since it has inverse $G(z, w) \mapsto G\left(w^{-k} z, w\right)$. This implies that

$$
I_{\mathbf{1}}(p, \tilde{p})=I_{\mathbf{1}}\left(\phi_{k}(p), \phi_{k}(\tilde{p})\right)
$$

This applies to $p \pm v$ as well.

Since $p$ is scattering stable, so is $\phi_{k}(p)$. Indeed, $\phi_{k}(p)$ has no zeros in $\mathbb{D}^{2}$ since $(z, w) \mapsto$ $\left(w^{k} z, w\right)$ maps the bidisk into itself. Also,

$$
\widetilde{\phi_{k}(p)}=z^{n} w^{k n+m} \overline{p\left(1 / \bar{w}^{k} 1 / \bar{z}, 1 / \bar{w}\right)}=\phi_{k}(\tilde{p})
$$

If $\phi_{k}(p)$ and $\phi_{k}(\tilde{p})$ had a nontrivial common factor say $\phi_{k}(p)=g h_{1}, \phi_{k}(\tilde{p})=g h_{2}$ then

$$
p(z, w)=g\left(w^{-k} z, w\right) h_{1}\left(w^{-k} z, w\right) \quad \tilde{p}(z, w)=g\left(w^{-k} z, w\right) h_{2}\left(w^{-k} z, w\right) .
$$

One can then argue that $g\left(w^{-k} z, w\right)$ is either already a polynomial or there exists $N$ such that $w^{N} g\left(w^{-k} z, w\right), w^{-N} h_{1}\left(w^{-k} z, w\right), w^{-N} h_{2}\left(w^{-k} z, w\right)$ are polynomials. Either case implies $p$ and $\tilde{p}$ have a non-trivial common factor contrary to assumption.

The same arguments apply to $p \pm v$. Therefore, by Proposition 3.6

$$
I_{\{z=1\}}\left(\phi_{k}(p), \phi_{k}(\tilde{p})\right) \leq I_{\{z=1\}}\left(\phi_{k}(p) \pm \phi_{k}(v), \phi_{k}(\tilde{p}) \pm \phi_{k}(v)\right) .
$$

Finally, we can choose $k$ so that $\phi_{k}(p), \phi_{k}(\tilde{p})$ and $\phi_{k}(p) \pm \phi_{k}(v), \phi_{k}(\tilde{p}) \pm \phi_{k}(v)$ have a single common zero on the line $\{z=1\}$ namely $\mathbf{1}=(1,1)$. Then, the above intersection multiplicities count the multiplicity at just $\mathbf{1}$ and these agree with the multiplicities associated to $p, \tilde{p}$ and $p \pm v, \tilde{p} \pm v$ as mentioned in equation (5.1).

This proves

which proves Theorem 1.6.

$$
I_{\mathbf{1}}(p, \tilde{p}) \leq I_{\mathbf{1}}(p \pm v, \tilde{p} \pm v)
$$

A corollary of the proof is the following fundamental fact.

Corollary 5.1. If $p \in \mathbb{C}[z, w]$ is scattering stable then for $\zeta \in \mathbb{T}^{2}, I_{\zeta}(p, \tilde{p})$ is even.

This follows because the intersection multiplicities in Proposition 3.6 are even. We gave a detailed proof of this corollary in Appendix $\mathrm{C}$ of [10].

\section{Degree $(1,1)$ polynomials}

Proof of Theorem 1.8. Suppose $p$ is scattering stable, has degree $(1,1)$, and $f=(p+\tilde{p}) /(p-\tilde{p})$ is an extreme point.

We can normalize so that $|p(0)|=1$. (We cannot normalize so that $p(0)=1$ because $p / p(0)$ has reflection $\tilde{p} / \overline{p(0)}$ not $\tilde{p} / p(0)$.)

Since $\tilde{p}(0)=0, p$ has the form $p(z, w)=\mu+a z+b w ; \mu \in \mathbb{T}, a, b, \in \mathbb{C}$. We can apply a rotation in each of the variables and replace $p$ with the polynomial $\mu(1-|a| z-|b| w)$; we will drop the absolute values on $a, b$ and assume $a, b>0$. This change of variables does not change extremality.

For $p$ to be stable we must have $1 \geq a+b$. Since $f$ is extreme, $p$ must have a zero on $\mathbb{T}^{2}$. This forces $1=a+b$ and the zero is $(z, w)=(1,1)$. Zeros on $\mathbb{T}^{2}$ occur with even multiplicity 
and this automatically implies $p$ is $\mathbb{T}^{2}$-saturated. (Thus, in this low degree situation a single zero forces the polynomial to be saturated.)

We need to show $p-\tilde{p}$ is irreducible. We have

$$
p-\tilde{p}=\mu+(\bar{\mu} b-\mu a) z+(\bar{\mu} a-\mu b) w-\bar{\mu} z w .
$$

If this were reducible it would factor into $\mu(1-c z)(1-d w)$. We will omit the details but the only way this can happen is if $c=d=1$ and $\mu= \pm i$. We can normalize so $\mu=i$. Then,

$$
p-\tilde{p}=i(1-(z+w)+z w)
$$

while

$$
p+\tilde{p}=i(1+t(z-w)-z w)
$$

where $t=b-a$. Then, one can directly check

$$
f=b \frac{1+z}{1-z}+a \frac{1+w}{1-w}
$$

contradicting extremality of $f$.

The next theorem is an amusing consequence of the above proof.

Theorem 6.1. Let $p \in \mathbb{C}\left[z_{1}, z_{2}\right]$ be scattering stable, $\tilde{p}(0,0)=0$, and $\operatorname{deg} p=(1,1)$. Set $F=\tilde{p} / p$ and for $\nu \in \mathbb{T}$,

$$
f_{\nu}=\frac{1+\nu^{2} F}{1-\nu^{2} F}=\frac{\bar{\nu} p+\nu \tilde{p}}{\bar{\nu} p-\nu \tilde{p}} .
$$

Then, there exists $\nu \in \mathbb{T}$ such that $f_{\nu}$ is not an extreme point of $\mathrm{PRP}_{2}$.

I. Klep and J. Pascoe have found an example which shows this theorem does not hold more generally.

Proof. Let $p \in \mathbb{C}[z, w]$ be scattering stable, $\operatorname{deg} p=(1,1)$, and $\tilde{p}(0,0)=0$. We claim some $f_{\nu}$ as in the theorem statement is not extreme. By Theorem 1.8 if $p$ is not $\mathbb{T}^{2}$-saturated, then $f_{\mu}$ is never extreme. So, we assume $p$ is $\mathbb{T}^{2}$-saturated.

If we follow the proof of Theorem 1.8 above, we see that if we set $\nu=i \mu$ then $\bar{\nu} p-\nu \tilde{p}=$ $i(1-z)(1-w)$ which means $f_{\nu}$ is not extreme.

\section{EXAMPLE OF A NON-SATURATED POLYNOMIAL}

Let $p(z, w)=3-z-z^{2}-w$ which is scattering stable, has degree $(2,1)$, and has a single zero on $\mathbb{T}^{2}$ at $(z, w)=(1,1)$. The multiplicity of this zero is 2 , so $p$ is not saturated (which would require 4 boundary zeros counting multiplicity). One can compute that this multiplicity is 2 using the resultant (with respect to $w$ ) of $p$ and $\tilde{p}=-z^{2}-w-z w+3 z^{2} w$ which is

$$
\operatorname{det}\left(\begin{array}{cc}
3-z-z^{2} & -1 \\
-z^{2} & -1-z+3 z^{2}
\end{array}\right)=-(z-1)^{2}\left(3 z^{2}+8 z+3\right) .
$$

Next, we claim that $f=\frac{p+\tilde{p}}{p-\tilde{p}}$ is not extreme. To do this we must perturb $p$ with $v$ satisfying $v=\tilde{v}, v(0,0)=0$, and stay stable. Let $v(z, w)=(1-z)(z-w)$. We claim $p+t v$ has no zeros in $\mathbb{D}^{2}$ for $t \in[-7 / 4,1 / 2]$. By the Schur-Cohn test we check when the following expression is non-negative on $\mathbb{T}$

$$
\left|3-z-z^{2}+t(1-z) z\right|^{2}-\left|-z^{2}+t(1-z) z\right|^{2} .
$$


This can be rewritten as

$$
|1-z|^{2}(2 t+8+6(t+1) \operatorname{Re} z)
$$

which is non-negative on $\mathbb{T}$ for $t \in[-7 / 4,1 / 2]$. Thus, for such $t, p+t v$ has no zeros in $\mathbb{T} \times \overline{\mathbb{D}}$ except for finitely many points on $\mathbb{T}^{2}$ and finitely many vertical lines by Lemma 3.4. We can rule out vertical lines since

$$
p-\tilde{p}=3-z+\left(3 z-z^{2}\right) w
$$

is irreducible (and if $p+t v$ had a factor $z-z_{0}$ with $z_{0} \in \mathbb{T}$ then so would $\tilde{p}+t v$ and so would $p-\tilde{p}$ ). By Lemma $3.3 p+t v$ has no zeros in $\mathbb{T} \times \mathbb{D}$. For $w \in \mathbb{D}$, the number of zeros of $z \mapsto(p+t v)(z, w)$ in $\mathbb{D}$ depends continuously on $w$ by the argument principle and hence must be constant. We can show $(p+t v)(z, 0)=3+(1-t) z-(1+t) z^{2}$ has no zeros in $\mathbb{D}$ directly by noting $|t-1|+|t+1| \leq 3$ for $t \in[-7 / 4,1 / 2]$. Therefore, $p+t v$ has no zeros in $\mathbb{D}^{2}$ for $t \in[-7 / 4,1 / 2]$. This proves $f$ is not extreme.

The endpoints of the line segment $q_{-}=p-7 v / 4$ and $q_{+}=p+v / 2$ are

$$
\begin{aligned}
& q_{-}=3-(11 / 4) z+(3 / 4) w-(3 / 4) z^{2}-(7 / 4) z w \\
& q_{+}=3-(1 / 2) z-(3 / 2) w-(3 / 2) z^{2}+(1 / 2) z w .
\end{aligned}
$$

Now, $q_{-}$has two zeros on $\mathbb{T}^{2} ;(1,1),(-1,1)$. Each has multiplicity 2 which implies $q_{-}$is $\mathbb{T}^{2}$-saturated. Since $p-\tilde{p}=q_{-}-\tilde{q_{-}}$is irreducible we conclude that $f_{-}=\frac{q_{-}+\tilde{q}_{-}}{q_{-} \tilde{q}_{-}}$is an extreme point by Theorem 1.5.

Next, $q_{+}$has a single zero on $\mathbb{T}^{2} ;(1,1)$. It occurs with multiplicity 4 which implies $q_{+}$is $\mathbb{T}^{2}$-saturated. As with $q_{-}$, we see that the analogue of $f_{-}$, namely $f_{+}$, is also an extreme point.

Then,

$$
f=(2 / 9) f_{-}+(7 / 9) f_{+}
$$

expresses $f$ as a convex combination of extreme points.

\section{Integrability OF PERTURBATIONS}

In this section we prove Theorem 1.9. We need to use several definitions and results from [10].

Definition 8.1. If $p \in \mathbb{C}[z, w]$ is scattering stable and $\vec{A}_{1} \in \mathbb{C}^{N}[z, w], \vec{A}_{2} \in \mathbb{C}^{M}[z, w]$ are vector polynomials satisfying

$$
|p|^{2}-|\tilde{p}|^{2}=\left(1-|z|^{2}\right)\left|\vec{A}_{1}\right|^{2}+\left(1-|w|^{2}\right)\left|\vec{A}_{2}\right|^{2}
$$

then we call $\left(\vec{A}_{1}, \vec{A}_{2}\right)$ an Agler pair.

For this definition, $N, M$ need not have any relation to the bidegree $(n, m)$ of $p$. One can in fact show $N \geq n, M \geq m$, but this is not important for now. Later we do look at Agler pairs with minimal dimensions.

It is proven in 2, 9] that $p$ has at least one Agler pair. (These vector polynomials are closely related to so-called Agler kernels for more general bounded analytic functions. See [1].)

Lemma 7.3 of [10] proves that the values of $\left(\left|\vec{A}_{1}\right|,\left|\vec{A}_{2}\right|\right)$ on $\mathbb{T}^{2}$ are the same for all Agler pairs. Therefore, the following proposition does not depend on the particular Agler pair. 
Proposition 8.2. Suppose $p \in \mathbb{C}[z, w]$ is scattering stable and $\left(\overrightarrow{A_{1}}, \overrightarrow{A_{2}}\right)$ is an Agler pair for $p$.

Given $q \in \mathbb{C}[z, w], q / p \in L^{2}\left(\mathbb{T}^{2}\right)$ if and only if there is a constant $C$ such that

$$
|q| \leq C\left(\left|\overrightarrow{A_{1}}\right|+\left|\overrightarrow{A_{2}}\right|\right)
$$

holds on $\mathbb{T}^{2}$.

Proof. Lemma 7.3 of [10] proves explicit formulas for $\left|\overrightarrow{A_{1}}\right|,\left|\overrightarrow{A_{2}}\right|$ on $\mathbb{T}^{2}$ in terms of $p$. Corollary 7.4 of [10] proves that the given inequality is equivalent to $q / p \in L^{2}\left(\mathbb{T}^{2}\right)$ however it is stated using said explicit formulas from Lemma 7.3 (so that the corollary does not need to reference Agler pairs).

The first part of Theorem 1.9 is contained in the following proposition.

Proposition 8.3. Suppose $p$ is scattering stable, $v=\tilde{v}$, and $p \pm v$ are scattering stable. Let $q \in \mathbb{C}[z, w]$. If $\frac{q}{p+v} \in L^{2}\left(\mathbb{T}^{2}\right)$ then $\frac{q}{p} \in L^{2}\left(\mathbb{T}^{2}\right)$. In particular, $v / p \in L^{2}\left(\mathbb{T}^{2}\right)$.

Proof. We can rescale $v$ so that $p \pm v$ are scattering stable. Let $\left(E_{ \pm}, F_{ \pm}\right)$be Agler pairs for $p \pm v$. Since

$$
\begin{aligned}
|p \pm v|^{2}-|\tilde{p} \pm v|^{2} & =|p|^{2}-|\tilde{p}|^{2} \pm 2 \operatorname{Re}(\overline{p-\tilde{p}} v) \\
& =\left(1-|z|^{2}\right)\left|E_{ \pm}\right|^{2}+\left(1-|w|^{2}\right)\left|F_{ \pm}\right|^{2}
\end{aligned}
$$

it follows that for $E=\frac{1}{\sqrt{2}}\left(\begin{array}{c}E_{+} \\ E_{-}\end{array}\right)$and $F=\left(\begin{array}{l}F_{+} \\ F_{-}\end{array}\right)$, the pair $(E, F)$ is an Agler pair for $p$. This comes from averaging the above formula over + and - .

Note that $|E| \geq \frac{1}{\sqrt{2}}\left|E_{ \pm}\right|,|F| \geq \frac{1}{\sqrt{2}}\left|F_{ \pm}\right|$on $\mathbb{T}^{2}$. These bounds along with Prop 8.2 automatically give that $q /(p+v) \in L^{2}\left(\mathbb{T}^{2}\right)$ implies $q / p \in L^{2}\left(\mathbb{T}^{2}\right)$ for $q \in \mathbb{C}[z, w]$.

Since $1=(p+v) /(p+v) \in L^{2}\left(\mathbb{T}^{2}\right)$ we conclude that $(p+v) / p \in L^{2}$ and hence $v / p \in L^{2}$.

For any scattering stable polynomial $p \in \mathbb{C}[z, w]$ with degree at most $(n, m)$ define the subspace

$$
K_{p}=\left\{q \in \mathbb{C}[z, w]: \operatorname{deg} q \leq(n-1, m-1) \text { and } q / p \in L^{2}\left(\mathbb{T}^{2}\right)\right\} .
$$

Theorem B of [10] states that

$$
\operatorname{dim} K_{p}=n m-\frac{1}{2} I_{\mathbb{T}^{2}}(p, \tilde{p})
$$

where $I_{\mathbb{T}^{2}}(p, \tilde{p})$ is the number of common zeros of $p$ and $\tilde{p}$ on $\mathbb{T}^{2}$ counted with multiplicities. We can then conclude the following.

Corollary 8.4. Under the assumptions of Proposition 8.3 we have

$$
\operatorname{dim} K_{p} \geq \operatorname{dim} K_{p+v}
$$

This also follows from Theorem 1.6. As mentioned in the introduction $p$ is $\mathbb{T}^{2}$-saturated if and only if $K_{p}=\{0\}$ (see Corollary 6.5 of [10]). We use this fact in the proof of the second half of Theorem 1.9 which is stated in the following proposition.

Proposition 8.5. Suppose $p \in \mathbb{C}[z, w]$ is scattering stable and not $\mathbb{T}^{2}$-saturated. Then, there exists nonzero $v \in \mathbb{C}[z, w]$ with $\operatorname{deg} v \leq(n, m), v=\tilde{v}, v(0,0)=0$ and $v / p \in L^{2}\left(\mathbb{T}^{2}\right)$. 
Proof. Since $p$ is not $\mathbb{T}^{2}$-saturated, there exists $f \in K_{p}, f \neq 0$. We can assume $z$ does not divide $f$ because otherwise $f / z \in K_{p}$ and we could divide out all of the factors of $z$ if necessary.

Let $\tilde{f}$ be the reflection of $f$ at degree $(n-1, m-1)$. Set $v=w f+z \tilde{f}$. Note $v$ is nonzero because $z$ does not divide $f$. Since $|f|=|\tilde{f}|$ on $\mathbb{T}^{2}$, we have $\tilde{f} / p \in L^{2}\left(\mathbb{T}^{2}\right)$. Therefore, $v / p \in L^{2}\left(\mathbb{T}^{2}\right)$. The other properties of $v$ are straightforward.

\section{Determinantal REPRESEntations AND TRANSFER FUNCTION REALIZATIONS}

In this section we discuss a number of formulas which are known to hold for scattering stable $p$ and corresponding $F=\tilde{p} / p$ in the case of two variables. When $p$ is saturated it turns out the formulas have a special form. The techniques of this section are well-known and this section is more of a supplement to the paper. See [7,8] for related results.

It is a well-established consequence of the sums of squares formula(1.4) that if $p \in \mathbb{C}\left[z_{1}, z_{2}\right]$ is scattering stable of bidegree $n=\left(n_{1}, n_{2}\right)$ then there exists a $(1+|n|) \times(1+|n|)$ unitary $U=\left(\begin{array}{ll}A & B \\ C & D\end{array}\right)$ where $A$ is $1 \times 1$ such that

$$
F(z)=A+B P(z)(1-D P(z))^{-1} C
$$

Here

$$
P(z)=\left(\begin{array}{cc}
z_{1} I_{n_{1}} & 0 \\
0 & z_{2} I_{n_{2}}
\end{array}\right)
$$

We call the formula (9.1) a unitary transfer function realization of $F$.

Since $F$ has denominator $p$, we see that $p$ divides $\operatorname{det}(I-D P(z))$. But, $\operatorname{det}(I-D P(z))$ has bidegree at most $n$ we must have $p(z)=p(0) \operatorname{det}(I-D P(z))$. Thus, $p$ has a contractive determinantal representation; so-called because $D$ is a contractive matrix. The matrix $D$ is not just contractive; it is also a rank one perturbation of a unitary matrix. Indeed, the matrices

$$
V_{\alpha}=D+\frac{\alpha}{1-\alpha A} C B
$$

are unitary for $\alpha \in \mathbb{T}$. This follows from

$$
U\left(\begin{array}{c}
\frac{\alpha}{1-\alpha A} B \\
I_{|n|}
\end{array}\right)=\left(\begin{array}{c}
\frac{1}{1-\alpha A} B \\
V_{\alpha}
\end{array}\right)
$$

which implies

$$
I-V_{\alpha}^{*} V_{\alpha}=\frac{1-|\alpha|^{2}}{|1-\alpha A|^{2}} B^{*} B
$$

and this is zero when $|\alpha|=1$.

Notice now that the numerator of $F(z)$ is

$$
\tilde{p}(z)=p(0) \operatorname{det}(I-D P(z)) F(z)=p(0)(A \operatorname{det}(I-D P(z))+B P(z) \operatorname{adj}(1-D P(z)) C)
$$

where adj denotes the classical adjoint or adjugate matrix. Observe that

$$
\begin{aligned}
p-\alpha \tilde{p} & =p(0)((1-\alpha A) \operatorname{det}(I-D P(z))-\alpha B P(z) \operatorname{adj}(I-D P(z)) C) \\
& =p(0)(1-\alpha A) \operatorname{det}\left(I-D P(z)-\frac{\alpha}{1-\alpha A} C B P(z)\right) \\
& =p(0)(1-\alpha A) \operatorname{det}\left(I-V_{\alpha} P(z)\right) .
\end{aligned}
$$


The second line used the "rank one matrix update formula"

$$
\operatorname{det}\left(M+x y^{*}\right)=\operatorname{det} M+y^{*} \operatorname{adj}(M) x .
$$

We conclude that $p-\alpha \tilde{p}$ has a unitary determinantal representation whenever $\alpha \in \mathbb{T}$. This gives a general way to describe the denominators of elements of $P R P R_{2}$.

The matrix $U$ can be chosen with special structure when $p$ is saturated.

Theorem 9.1. If $p \in \mathbb{C}\left[z_{1}, z_{2}\right]$ is $\mathbb{T}^{2}$-saturated, then $F=\frac{\tilde{p}}{p}$ possesses a symmetric unitary transfer function realization; i.e. $U$ in (9.1) can be chosen to be a unitary and symmetric: $U^{*}=U^{-1}$ and $U=U^{t}$. In particular, $p$ has a determinantal representation using a symmetric contraction (which is a rank one perturbation of a symmetric unitary) and $p-\tilde{p}$ has a symmetric unitary determinantal representation.

This theorem can be subdivided into two parts.

Proposition 9.2. If $p \in \mathbb{C}\left[z_{1}, z_{2}\right]$ is $\mathbb{T}^{2}$-saturated with $\operatorname{deg} p=\left(n_{1}, n_{2}\right)$ then there exist $E_{1} \in \mathbb{C}^{n_{1}}\left[z_{1}, z_{2}\right], E_{2} \in \mathbb{C}^{n_{2}}\left[z_{1}, z_{2}\right]$ of bidegrees at most $\left(n_{1}-1, n_{2}\right),\left(n_{1}, n_{2}-1\right)$ such that

$$
|p(z)|^{2}-|\tilde{p}(z)|^{2}=\left(1-\left|z_{1}\right|^{2}\right)\left|E_{1}(z)\right|^{2}+\left(1-\left|z_{2}\right|^{2}\right)\left|E_{2}(z)\right|^{2}
$$

and

$$
E_{1}(z)=\underbrace{z_{1}^{n_{1}-1} z_{2}^{n_{2}} \overline{E_{1}\left(1 / \bar{z}_{1}, 1 / \bar{z}_{2}\right)}}_{:=\tilde{E}_{1}} \quad E_{2}(z)=\underbrace{z_{1}^{n_{1}} z_{2}^{n_{2}-1} \overline{E_{2}\left(1 / \bar{z}_{1}, 1 / \bar{z}_{2}\right)}}_{:=\tilde{E}_{2}} .
$$

This follows directly from Theorem 1.1.5 of [9] and Corollary 13.6 of [10. The condition in (9.4) can be replaced with the weaker conditions $\left|E_{1}\right|=\left|\tilde{E}_{1}\right|,\left|E_{2}\right|=\left|\tilde{E}_{2}\right|$. (An argument is given in the proof of Theorem 1.1.5 of [9]. It uses the fact that any symmetric unitary $U$ can be factored as $U=V^{t} V$ where $V$ is unitary.)

Proposition 9.3. If $p$ is scattering stable and possesses a sums of squares decomposition as in (9.3) which in addition satisfies (9.4), then $F=\frac{\tilde{p}}{p}$ satisfies (9.1) where $U$ may be chosen to be symmetric.

Symmetry in the sums of squares formulas does not characterize saturated polynomials.

Example 9.4. Let $p=\left(3-z_{1}-z_{2}\right)^{2}$-a non-saturated polynomial. Then, $\tilde{p}=\left(3 z_{1} z_{2}-z_{1}-\right.$ $\left.z_{2}\right)^{2}$ and (9.3) holds with

$$
E_{1}=\left(\begin{array}{l}
q\left(z_{2}\right) p(z) \\
\tilde{q}\left(z_{2}\right) \tilde{p}(z)
\end{array}\right) \quad E_{2}=\left(\begin{array}{l}
\tilde{q}\left(z_{1}\right) p(z) \\
q\left(z_{1}\right) \tilde{p}(z)
\end{array}\right)
$$

where $q(\zeta)=\sqrt{3}\left(\frac{1-\sqrt{5}}{2}+\frac{1+\sqrt{5}}{2} \zeta\right)$ and $\tilde{q}$ is the reflection at degree 1 of this one variable polynomial. Evidently, $\left|E_{j}\right|=\left|\tilde{E}_{j}\right|$.

It may be possible to use the characterization of all sums of squares decompositions (9.3) given in [10] to characterize which $p$ have decompositions satisfying (9.4).

We now prove Proposition 9.3. Unfortunately, the only way we see to give a comprehensible proof is to rehash a number of standard arguments (including the proof of (9.1)). 
Proof of Proposition 9.3. Initially we do not need (9.4).

Equation (9.3) can be polarized to the following form

$$
\overline{p(w)} p(z)-\overline{\tilde{p}(w)} \tilde{p}(z)=\left(1-\bar{w}_{1} z_{1}\right) E_{1}(w)^{*} E_{1}(z)+\left(1-\bar{w}_{2} z_{2}\right) E_{2}(w)^{*} E_{2}(z)
$$

by the polarization theorem for holomorphic functions. We now use what is called a lurking isometry argument. Let $N=1+n_{1}+n_{2}$. Define

$$
X(z):=\left(\begin{array}{c}
p(z) \\
z_{1} E_{1}(z) \\
z_{2} E_{2}(z)
\end{array}\right) \quad Y(z):=\left(\begin{array}{c}
\tilde{p}(z) \\
E_{1}(z) \\
E_{2}(z)
\end{array}\right) .
$$

Then, (9.5) rearranges into $X(w)^{*} X(z)=Y(w)^{*} Y(z)$. The map

$$
X(z) \mapsto Y(z)
$$

extends to a well-defined linear isometry from the span of the elements on the left to the span of the elements on the right (as $z$ varies over $\mathbb{C}^{2}$ ). It turns out that $\operatorname{span}\left\{X(z): z \in \mathbb{C}^{2}\right\}=\mathbb{C}^{N}$ and this implies the map above actually extends uniquely to a unitary. This follows from the construction of $E_{1}, E_{2}$ in [9]. However, as this fact is difficult to retrieve from [9] without introducing too much machinery we proceed without it. The map (9.6) can always be extended to some unitary; we would like to show that if $E_{1}, E_{2}$ satisfy (9.4) then we can extend to a symmetric unitary.

So, suppose (9.4) holds and notice that $Y=\tilde{X}$, with the reflection performed at the degree $\left(n_{1}, n_{2}\right)$. We introduce the orthogonal complements of the spans of the left and right sides in (9.6)

$$
\begin{aligned}
& S_{1}=\operatorname{span}\left\{X(z): z \in \mathbb{C}^{2}\right\}^{\perp} \\
& S_{2}=\operatorname{span}\left\{\tilde{X}(z): z \in \mathbb{C}^{2}\right\}^{\perp}
\end{aligned}
$$

Notice that $v \in S_{1}$ iff $\bar{v} \in S_{2}$ because we can reflect the equation $X^{*} v=0$ to obtain $\tilde{X}^{*} \bar{v}=0$. Let $v_{1}, \ldots, v_{k}$ be an orthonormal basis for $S_{1}$. Then, $\bar{v}_{1}, \ldots, \bar{v}_{k}$ is an orthonormal basis for $S_{2}$. We can then extend the map (9.6) to all of $\mathbb{C}^{N}$ by mapping $v_{j} \mapsto \bar{v}_{j}$ and extending linearly. Note that we are not saying $v \mapsto \bar{v}$ for a general $v \in S_{1}$. We get an $N \times N$ unitary matrix $U$ with the property

$$
U X(z)=\tilde{X}(z) \text { and } U v_{j}=\bar{v}_{j} .
$$

Applying the reflection operation at the degree $\left(n_{1}, n_{2}\right)$ and conjugation reveals

$$
U^{t} X(z)=\tilde{X}(z) \text { and } U^{t} v_{j}=\bar{v}_{j} \text {. }
$$

Therefore, $U=U^{t}$.

Write $U=\left(\begin{array}{ll}A & B \\ C & D\end{array}\right)$ where $A$ is $1 \times 1$; the sizes of $B, C, D$ are then determined. Letting $E=\left(\begin{array}{l}E_{1} \\ E_{2}\end{array}\right)$ we have

$$
\begin{aligned}
& A p(z)+B P(z) E(z)=\tilde{p} \\
& C p(z)+D P(z) E(z)=E(z)
\end{aligned}
$$

where $P(z)$ is given in (9.2). The second line implies $p(z)(I-D P(z))^{-1} C=E(z)$ which implies $A+B P(z)(I-D P(z))^{-1} C=\tilde{p} / p$ via the first line. 


\section{ACKNOWLEDGMents}

I would like to thank Mike Jury, John McCarthy, and James Pascoe for useful discussions. Thanks to Pascoe and Klep for disproving a conjectured generalization of Theorem 6.1.

\section{REFERENCES}

[1] Kelly Bickel and Greg Knese, Canonical Agler decompositions and transfer function realizations, Trans. Amer. Math. Soc. 368 (2016), no. 9, 6293-6324, DOI 10.1090/tran/6542. MR3461035

[2] Brian J. Cole and John Wermer, Ando's theorem and sums of squares, Indiana Univ. Math. J. 48 (1999), no. 3, 767-791, DOI 10.1512/iumj.1999.48.1716. MR1736979

[3] Gerd Fischer, Plane algebraic curves, Student Mathematical Library, vol. 15, American Mathematical Society, Providence, RI, 2001. Translated from the 1994 German original by Leslie Kay. MR1836037

[4] William Fulton, Algebraic curves, Advanced Book Classics, Addison-Wesley Publishing Company, Advanced Book Program, Redwood City, CA, 1989. An introduction to algebraic geometry; Notes written with the collaboration of Richard Weiss; Reprint of 1969 original. MR1042981

[5] Frank Forelli, A necessary condition on the extreme points of a class of holomorphic functions. II, Pacific J. Math. 92 (1981), no. 2, 277-281. MR618065 (82k:32021)

[6] Jeffrey S. Geronimo and Hugo J. Woerdeman, Positive extensions, Fejér-Riesz factorization and autoregressive filters in two variables, Ann. of Math. (2) 160 (2004), no. 3, 839-906, DOI 10.4007/annals.2004.160.839. MR2144970

[7] Anatolii Grinshpan, Dmitry S. Kaliuzhnyi-Verbovetskyi, Victor Vinnikov, and Hugo J. Woerdeman, Stable and real-zero polynomials in two variables, Multidimens. Syst. Signal Process. 27 (2016), no. 1, 1-26, DOI 10.1007/s11045-014-0286-3. MR3441374

[8] Greg Knese, Polynomials defining distinguished varieties, Trans. Amer. Math. Soc. 362 (2010), no. 11, 5635-5655, DOI 10.1090/S0002-9947-2010-05275-4. MR2661491

[9] _ Polynomials with no zeros on the bidisk, Anal. PDE 3 (2010), no. 2, 109-149, DOI 10.2140/apde.2010.3.109. MR2657451

[10] _ Integrability and regularity of rational functions, Proc. Lond. Math. Soc. (3) 111 (2015), no. 6, 1261-1306, DOI 10.1112/plms/pdv061. MR3447794

[11] Peter Lancaster and Miron Tismenetsky, The theory of matrices, 2nd ed., Computer Science and Applied Mathematics, Academic Press, Inc., Orlando, FL, 1985. MR792300

[12] John N. McDonald, Holomorphic functions on the polydisc having positive real part, Michigan Math. J. 34 (1987), no. 1, 77-84, DOI 10.1307/mmj/1029003484. MR873021 (88b:32004)

[13] _ An extreme absolutely continuous RP-measure, Proc. Amer. Math. Soc. 109 (1990), no. 3, 731-738, DOI 10.2307/2048213. MR1017849 (90k:32005)

[14] Vlastimil Pták and N. J. Young, A generalization of the zero location theorem of Schur and Cohn, IEEE Trans. Automat. Control 25 (1980), no. 5, 978-980, DOI 10.1109/TAC.1980.1102476. MR595237

[15] Walter Rudin, Function theory in polydiscs, W. A. Benjamin, Inc., New York-Amsterdam, 1969. MR0255841

[16] _ Harmonic analysis in polydiscs, Actes du Congrès International des Mathématiciens (Nice, 1970), Gauthier-Villars, Paris, 1971, pp. 489-493. MR0422657

Washington University in St. Louis, Department of Mathematics, St. Louis, MO 63130 E-mail address: geknese@wustl.edu 